\title{
Towards gravitationally assisted negative refraction of light by vacuum
}

\author{
Akhlesh Lakhtakia* \\ CATMAS - Computational \& Theoretical Materials Sciences Group \\ Department of Engineering Science and Mechanics \\ Pennsylvania State University, University Park, PA 16802-6812, USA \\ Tom G. Mackay ${ }^{\dagger}$ \\ School of Mathematics, University of Edinburgh, Edinburgh EH9 3JZ, UK
}

\begin{abstract}
Propagation of electromagnetic plane waves in some directions in gravitationally affected vacuum over limited ranges of spacetime can be such that the phase velocity vector casts a negative projection on the time-averaged Poynting vector. This conclusion suggests, inter alia, gravitationally assisted negative refraction by vacuum.
\end{abstract}

Keywords: General theory of relativity, Gravitational lensing, Hidden matter, Negative phase velocity, Poynting vector, Special theory of relativity

\section{Introduction}

The discovery of (purportedly) isotropic, homogeneous, dielectric-magnetic materials that bend electromagnetic rays the "wrong way" [1] created quite a stir in 2001 [2], with claims and counterclaims flying all around [3, 4]. The situation has recently been settled, with unequivocal demonstrations by several independent groups [5]-[7]. See Ref. 8 for a comprehensive review. A range of exotic and potentially useful phenomenons - such as negative refraction, negative Doppler shift and inverse Cerenkov radiation - have been predicted for materials of this type, wherein the phase velocity is directed in opposition to the energy velocity as quantified through the time-averaged Poynting vector. These materials have several names, including left-handed materials, negative-index materials, and negative-phasevelocity (NPV) materials. We prefer the last term [8].

Subsequently, the possibility of NPV propagation of light and other electromagnetic waves was established in a variety of anisotropic materials [9]-[11]. In these materials, NPV propagation is indicated by the projection of the phase velocity on the time-averaged Poynting vector being negative.

\footnotetext{
*Fax: +1 814863 4319; e-mail: akhlesh@psu.edu; also affiliated with Department of Physics, Imperial College, London SW7 2 BZ, UK

${ }^{\dagger}$ Corresponding Author. Fax: + 44131650 6553; e-mail: T.Mackay@ed.ac.uk.
} 
Even more interestingly, materials that do not permit the observation of NPV propagation by observers in a relatively stationary (i.e., co-moving) inertial reference frame have been shown, after the invocation of the postulates of special theory of relativity (STR), to allow observation of NPV propagation in other inertial frames [12]. That permits one to envisage STR negative refraction being exploited in astronomical scenarios such as, for example, in the remote sensing of planetary and asteroidal surfaces from space stations. Quite possibly, space telemetry technologies will be the first to reap the benefits of STR negative refraction. Application to remotely guided, extraterrestrial mining and manufacturing industries can also be envisioned. Furthermore, many unusual astronomical phenomenons would be discovered and/or explained via STR negative refraction to interpret data collected via telescopes [13].

As is well known, vacuum (i.e., matter-free space) appears the same to all inertial observers [14]. Therefore, as a co-moving observer cannot deduce the occurrence of NPV propagation in vacuum, neither can any observer moving with a constant velocity. This could lead one to believe that NPV propagation is impossible in huge expanses of interstellar space. However, gravitational fields from nearby massive objects will certainly distort electromagnetic propagation, which is a principal consequence of the general theory of relativity and is indeed used nowadays in GPS systems. Our objective here is to establish that gravitationally affected vacuum can support NPV propagation, at least in spacetime manifolds of limited extent.

\section{Theory}

A gravitational field curves spacetime, which effect is captured through a metric $g_{\alpha \beta} \cdot^{\ddagger}$ Electromagnetic propagation in gravitationally affected vacuum may be described in terms of propagation in an instantaneously responding medium in flat spacetime [15, 16], at least in spacetime manifolds of limited extent. That is, the nonuniform metric $g_{\alpha \beta}$ can be locally approximated by the uniform metric $\tilde{g}_{\alpha \beta}[17]$. On asssuming the convention $\tilde{g}_{\alpha \beta}=(+,-,-,-)$, the constitutive relations of vacuum in the equivalent flat spacetime are expressed in Gaussian units as [16]

$$
\begin{aligned}
D_{\ell} & =\epsilon_{\ell m} E_{m}+\epsilon_{\ell m n} \tilde{g}_{m} H_{n}, \\
B_{\ell} & =\mu_{\ell m} H_{m}-\epsilon_{\ell m n} \tilde{g}_{m} E_{n},
\end{aligned}
$$

where $\epsilon_{\ell m n}$ is the Levi-Civita tensor, and

$$
\begin{aligned}
\epsilon_{\ell m} & =\mu_{\ell m}=-(-\tilde{g})^{1 / 2} \frac{\tilde{g}^{\ell m}}{\tilde{g}_{00}}, \\
\tilde{g}_{\ell} & =\frac{\tilde{g}_{0 \ell}}{\tilde{g}_{00}}
\end{aligned}
$$

with $\tilde{g}=\operatorname{det}\left[\tilde{g}_{\alpha \beta}\right]$. We note that the metric $\tilde{g}_{\alpha \beta}$ is real symmetric [18].

\footnotetext{
${ }^{\ddagger}$ Roman indexes take the values 1,2 and 3 ; while Greek indexes take the values $0,1,2$, and 3 .
} 
The constitutive relations (1) and (2) can be expressed in 3-vector form as

$$
\begin{aligned}
& \underline{D}=\epsilon_{0} \underline{\underline{\gamma}} \cdot \underline{E}-\underline{\Gamma} \times \underline{H}, \\
& \underline{B}=\mu_{0} \underline{\underline{\gamma}} \cdot \underline{H}+\underline{\Gamma} \times \underline{E},
\end{aligned}
$$

wherein SI units are implemented. The scalar constants $\epsilon_{0}$ and $\mu_{0}$ denote the permittivity and permeability of vacuum in the absence of a gravitational field, respectively, and $c_{0}=\sqrt{1 / \epsilon_{0} \mu_{0}}$. Our coordinate system is chosen such that the second-rank cartesian tensor $\gamma$ is diagonal; i.e., $\underline{\gamma}=\operatorname{diag}\left(\gamma_{x}, \gamma_{y}, \gamma_{z}\right)$. In the gravitational field of a mass rotating with angular momentum $\underline{\bar{J}}$, $\overline{\bar{t}}$ he gyrotropic vector $\underline{\Gamma}$ is proportional to $\underline{R} \times \underline{J}$, where $\underline{R}$ is the radial vector from the centre of mass to the point of observation. By considering a small region of space at a sufficiently remote location from the centre of mass, we take $\underline{\Gamma}$ to be independent of $\underline{R}$.

We seek planewave solutions

$$
\begin{aligned}
& \underline{E}=\operatorname{Re}\left\{\underline{E}_{0} \exp [i(\underline{k} \cdot \underline{r}-\omega t)]\right\}, \\
& \underline{H}=\operatorname{Re}\left\{\underline{H}_{0} \exp [i(\underline{k} \cdot \underline{r}-\omega t)]\right\},
\end{aligned}
$$

to the source-free Maxwell curl postulates

$$
\begin{aligned}
& \nabla \times \underline{E}+\frac{\partial}{\partial t} \underline{B}=\underline{0}, \\
& \nabla \times \underline{H}-\frac{\partial}{\partial t} \underline{D}=\underline{0} .
\end{aligned}
$$

Here $\underline{k}$ is the wavevector, $\underline{r}$ is the position vector, $\omega$ is the angular frequency, and $t$ denotes the time; whereas $\underline{E}_{0}$ and $\underline{H}_{0}$ are complex-valued amplitudes.

An eigenvector equation for $\underline{E}_{0}$ is developed as follows. By combining (5)-(8) with the Maxwell curl postulates, we derive

$$
\begin{aligned}
& \underline{p} \times \underline{E}_{0}=\omega \mu_{0} \underline{\underline{\gamma}} \cdot \underline{H}_{0} \\
& \underline{p} \times \underline{H}_{0}=-\omega \epsilon_{0} \underline{\underline{\gamma}} \cdot \underline{E}_{0}
\end{aligned}
$$

in terms of

$$
\underline{p}=\underline{k}-\omega \underline{\Gamma} .
$$

The use of (11) to eliminate $\underline{H}_{0}$ from (12) provides, after some simplification,

$$
\underline{\underline{W}} \cdot \underline{E}_{0}=\underline{0}
$$

where

$$
\underline{\underline{W}}=\left(k_{0}^{2} \operatorname{det}[\underline{\gamma}]-\underline{p} \cdot \underline{\underline{\gamma}} \cdot \underline{p}\right) \underline{\underline{I}}+\underline{p} \underline{p} \cdot \underline{\underline{\gamma}},
$$

and the notation $k_{0}=\omega \sqrt{\epsilon_{0} \mu_{0}}$ has been introduced. A dispersion relation thus emerges from (14) as

$$
\operatorname{det}[\underline{\underline{W}}]=0 \text {, }
$$


which may be expressed in the form

$$
k_{0}^{2} \operatorname{det}[\underline{\gamma}]\left(k_{0}^{2} \operatorname{det}[\underline{\gamma}]-\underline{p} \cdot \underline{\gamma} \cdot \underline{p}\right)^{2}=0 .
$$

Hence, we conclude that planewave solutions satisfy the condition

$$
\underline{p} \cdot \underline{\underline{\gamma}} \cdot \underline{p}=k_{0}^{2} \operatorname{det}[\underline{\underline{\gamma}}] \text {. }
$$

Let us consider eigenvector solutions to (14). Substitution of (18) into (14) provides

$$
\underline{p} \underline{p} \cdot \underline{\gamma} \cdot \underline{E}_{0}=\underline{0}
$$

thereby, all eigenvector solutions are necessarily orthogonal to $\underline{p} \cdot \underline{\gamma}$. To proceed further, let us - without any loss of generality — choose the wavevector $\underline{k}$ to lie along $z$ axis, and the vector $\underline{\Gamma}$ to lie in the $x z$ plane; i.e.,

$$
\begin{aligned}
\underline{k} & =k \underline{\hat{u}}_{z}, \\
\underline{\Gamma} & =\Gamma\left(\underline{\hat{u}}_{x} \sin \theta+\underline{\hat{u}}_{z} \cos \theta\right),
\end{aligned}
$$

where $\underline{\hat{u}}_{x}, \underline{\hat{u}}_{y}$ and $\underline{\hat{u}}_{z}$ are the cartesian unit vectors in the equivalent flat spacetime. Since

$$
\underline{p} \cdot \underline{\underline{\gamma}}=-\omega \Gamma \gamma_{x} \sin \theta \underline{\hat{u}}_{x}+(k-\omega \Gamma \cos \theta) \gamma_{z} \underline{\hat{u}}_{z},
$$

it is clear that two linearly independent eigenvectors satisfying (18) may be stated as

$$
\begin{aligned}
\underline{e}_{1} & =\underline{\hat{u}}_{y}, \\
\underline{e}_{2} & =\underline{\hat{u}}_{y} \times(\underline{p} \cdot \underline{\underline{\gamma}}) \\
& =(k-\omega \Gamma \cos \theta) \gamma_{z} \underline{\hat{\hat{u}}}_{x}+\omega \Gamma \gamma_{x} \sin \theta \underline{\hat{u}}_{z} .
\end{aligned}
$$

After assuming that $\underline{\underline{\gamma}}$ is invertible, we deduce the corresponding magnetic field eigenvectors from (11) as

$$
\begin{aligned}
& \underline{h}_{1}=\frac{1}{\omega \mu_{0}} \underline{\gamma}^{-1} \cdot\left[(\omega \Gamma \cos \theta-k) \underline{\hat{u}}_{x}-\omega \Gamma \sin \theta \underline{\hat{u}}_{z}\right], \\
& \underline{h}_{2}=\frac{1}{\omega \mu_{0}} \underline{\gamma}^{-1} \cdot\left[(k-\omega \Gamma \cos \theta)^{2} \gamma_{z}+(\omega \Gamma \sin \theta)^{2} \gamma_{x}\right] \underline{\hat{u}}_{y} .
\end{aligned}
$$

Hence, the general solution is given by

$$
\begin{aligned}
\underline{E}_{0}= & C_{1} \underline{\hat{u}}_{y}+C_{2}\left[(k-\omega \Gamma \cos \theta) \gamma_{z} \underline{\hat{u}}_{x}+\omega \Gamma \gamma_{x} \sin \theta \underline{\hat{u}}_{z}\right], \\
\underline{H}_{0}= & \frac{1}{\omega \mu_{0}} \underline{\gamma}^{-1} \cdot\left\{C_{1}\left[(\omega \Gamma \cos \theta-k) \underline{\hat{u}}_{x}-\omega \Gamma \sin \theta \underline{\hat{u}}_{z}\right]\right. \\
& \left.+C_{2}\left[(k-\omega \Gamma \cos \theta)^{2} \gamma_{z}+(\omega \Gamma \sin \theta)^{2} \gamma_{x}\right] \underline{\hat{u}}_{y}\right\},
\end{aligned}
$$


wherein $C_{1}$ and $C_{2}$ are arbitrary constants.

The wavenumbers arise from the dispersion relation (17) as follows. Substituting (13) into (18), we obtain the $k$-quadratic expression

$$
k^{2} \gamma_{z}-2 k \gamma_{z} \omega \Gamma \cos \theta+\omega^{2} \Gamma^{2}\left(\gamma_{x} \sin ^{2} \theta+\gamma_{z} \cos ^{2} \theta\right)-k_{0}^{2} \operatorname{det}[\underline{\underline{\gamma}}]=0,
$$

since $\underline{k} \cdot \underline{\Gamma}=k \Gamma \cos \theta$. The two $k$-roots of $(30)$ are

$$
\begin{aligned}
& k^{+}=\omega\left(\Gamma \cos \theta+\sqrt{\epsilon_{0} \mu_{0} \gamma_{x} \gamma_{y}-\frac{\gamma_{x}}{\gamma_{z}} \Gamma^{2} \sin ^{2} \theta}\right), \\
& k^{-}=\omega\left(\Gamma \cos \theta-\sqrt{\epsilon_{0} \mu_{0} \gamma_{x} \gamma_{y}-\frac{\gamma_{x}}{\gamma_{z}} \Gamma^{2} \sin ^{2} \theta}\right) .
\end{aligned}
$$

Finally, let us consider the time-averaged Poynting vector given by

$$
\underline{P}=\frac{1}{2} \operatorname{Re}\left\{\underline{E}_{0} \times \underline{H}_{0}^{*}\right\}
$$

After utilizing the general solution (28) and (29), the component of the Poynting vector aligned with the $\underline{\hat{u}}_{z}$ axis is obtained as

$$
\underline{\hat{u}}_{z} \cdot \underline{P}=\frac{1}{2 \omega \mu_{0} \gamma_{z}}(k-\omega \Gamma \cos \theta)\left(\left|C_{1}\right|^{2}+\left|C_{2}\right|^{2} \gamma_{z} \omega^{2} \operatorname{det}[\underline{\underline{\gamma}}]\right) \text {. }
$$

The energy density flow in the direction of the wavevector $\underline{k}^{+}$, corresponding to the root $k^{+}$given in (31), is thus

$$
\begin{aligned}
\underline{k}^{+} \cdot \underline{P}= & \frac{1}{2 \mu_{0} \gamma_{z}}\left[\Gamma \cos \theta \sqrt{\epsilon_{0} \mu_{0} \gamma_{x} \gamma_{y}-\frac{\gamma_{x}}{\gamma_{z}} \Gamma^{2} \sin ^{2} \theta}+\left(\epsilon_{0} \mu_{0} \gamma_{x} \gamma_{y}-\frac{\gamma_{x}}{\gamma_{z}} \Gamma^{2} \sin ^{2} \theta\right)\right] \\
& \times\left(\left|C_{1}\right|^{2}+\left|C_{2}\right|^{2} \gamma_{z} \omega^{2} \operatorname{det}[\underline{\gamma}]\right) .
\end{aligned}
$$

Let us notice that the inequality

$$
\epsilon_{0} \mu_{0} \gamma_{x} \gamma_{y}-\frac{\gamma_{x}}{\gamma_{z}} \Gamma^{2} \sin ^{2} \theta \geq 0
$$

must be fulfilled in order for $k$ to be real-valued. Therefore, the defining inequality for NPV propagation, namely

$$
\underline{k}^{+} \cdot \underline{P}<0
$$

is satisfied provided that

$$
-\Gamma \cos \theta>\sqrt{\epsilon_{0} \mu_{0} \gamma_{x} \gamma_{y}-\frac{\gamma_{x}}{\gamma_{z}} \Gamma^{2} \sin ^{2} \theta}
$$

holds. Analogously, we find that NPV propagation is signalled for the $k^{-}$wavenumber by the condition

$$
\Gamma \cos \theta>\sqrt{\epsilon_{0} \mu_{0} \gamma_{x} \gamma_{y}-\frac{\gamma_{x}}{\gamma_{z}} \Gamma^{2} \sin ^{2} \theta}
$$

In deriving (38) and (39), we used the fact that $\gamma_{x, y, z}>0$ by virtue of the signature of $\tilde{g}_{\alpha \beta}$.

We note that the NPV conditions (38) and (39) are independent of frequency. 


\section{Concluding Remarks}

The inequalities (38) and (39) can be satisfied for specific ranges of the angle $\theta$, for given $\underline{\gamma}$ and $\Gamma$. Thus, we have shown that NPV propagation in some directions is possible in gravitationally affected vacuum over limited ranges of spacetime. The possible existence of gravitational fields which can deliver $\gamma$ and $\Gamma$ necessary for the satisfaction of (38) and/or (39) is a matter for astrophysicists to $\overline{\bar{d}}$ iscuss.

We are content here to state that, just as scientific and technological applications of STR negative refraction (by materials) can be envisaged $[12,13]$, similar and different consequences of gravitationally assisted negative refraction by vacuum are possible. In particular, designers of channels for space communication shall also have to account for the possibility of negative refraction due to massive objects between the two ends of every channel.

Furthermore, current ideas on the distribution of mass in the as-observed universe may require significant revision, since electromagnetic signals from distant objects may be deflected in a manner which has not hitherto been accounted for. Thus, our work has implications for gravitational lenses [19]. Gravitational lensing involves nonuniform metrics, and the distribution of matter in the universe has been constantly changing. While the spatiotemporally local evolution of the universe may be deduced adequately from electromagnetic signals received by our telescopes, reasonably accurate deductions about the spatiotemporally global evolution of the universe from similar measurements may be particularly difficult to make owing to gravitationally assisted negative refraction.

Finally, our work suggests that it is time that research on consequences of gravitationally assisted negative refraction by materials be undertaken.

Acknowledgement. We gratefully acknowledge discussions with Dr. Sandi Setiawan (University of Edinburgh), Dr. Chandra Roychoudhuri (University of Connecticut) and Mr. James McIlroy (Imperial College). TGM thanks the Nuffield Foundation for supporting his visit to Pennsylvania State University.

\section{References}

[1] http://plus.maths.org/issue15/news/refract/ (accessed on Aug 4, 2004)

[2] Shelby RA, Smith DR, and Schultz S 2001 Experimental verification of a negative index of refraction Science 292 77-79

[3] Garcia N and Nieto-Vesperinas M 2002 Is there an experimental verification of a negative index of refraction yet? Opt. Lett. 27 885-887

[4] Valanju PM, Walser RM and Valanju AP 2002 Wave refraction in negative-index media: always positive and very inhomogeneous Phys. Rev. Lett. 88187401

[5] Grbic A and Eleftheriades GV 2002 Experimental verification of backward-wave radiation from a negative index metamaterial J. Appl. Phys. 92 5930-5935 
[6] Houck AA, Brock JB and Chuang IL 2003 Experimental observations of a left-handed material that obeys Snell's law Phys. Rev. Lett. 90137401

[7] Pendry J B and Smith D R 2004 Reversing light with negative refraction Phys. Today 57(6), 37-43 (June issue)

[8] Lakhtakia A, McCall MW, and Weiglhofer WS 2003 Negative phase-velocity mediums Introduction to Complex Mediums for Optics and Electromagnetics ed WS Weiglhofer and A Lakhtakia (Bellingham, WA: SPIE Press)

[9] Hu L and Chui ST 2002 Characteristics of electromagnetic wave propagation in uniaxially anisotropic left-handed materials Phys. Rev. B 66085108

[10] Kärkkäinen MK 2003 Numerical study of wave propagation in uniaxially anisotropic Lorentzian backward-wave slabs Phys. Rev. E 68026602

[11] Mackay TG and Lakhtakia A 2004 Plane waves with negative phase velocity in Faraday chiral mediums Phys. Rev. E 69026602

[12] Mackay TG and Lakhtakia A 2004 Negative phase velocity in a uniformly moving, homogeneous, isotropic, dielectric-magnetic medium J. Phys. A: Math. Gen. 37 56975711

[13] Mackay TG and Lakhtakia A 2004 Negative refraction in outer space? Curr. Sci. 86 1593

[14] Chen HC 1983 Theory of Electromagnetic Waves (New York: McGraw-Hill) Chap 8

[15] Skrotskii GV 1957 The influence of gravitation on the propagation of light Soviet Phys.Dokl. 2 226-229

[16] Plebanski J 1960 Electromagnetic waves in gravitational fields Phys. Rev. 118, 13961408

[17] Foster J and Nightingale JD 1995 A Short Course in General Relativity, 2nd ed (New York: Springer) pp 4,121

[18] Sachs M 1993 Relativity in Our Time (London: Taylor \& Francis) p 114

[19] Koopmans L V E and Blandford R D 2004 Gravitational lenses Phys. Today 57(6), 45-51 (June issue) 